\title{
Optimum Energy Allocation for Massive Spread-Spectrum Multiple Access in Networks of
}

\author{
Uncoordinated Energy-Limited Terminals
}

\author{
Javier Villares, Francesc Rey and Josep Sala-Alvarez \\ Dept. of Signal Theory and Communications, Universitat Politècnica de Catalunya - Barcelona Tech, Spain \\ E-mail: \{javier.villares,francesc.rey,josep.sala\}@upc.edu ,WWW: http://spcom.upc.edu
}

\begin{abstract}
This paper derives the energy allocation policy that maximizes the aggregate spectral efficiency of an uncoordinated spread-spectrum multiple access network operating in the largeuser regime and subject to a long-term per-user average energy constraint. Focusing on a network of autonomous energy-limited devices, we consider that the receiver operates under noisedominant conditions and that every transmitter is able to estimate accurately its own slowly time-varying channel with no feedback from the receiver. The aggregate spectral efficiency is derived in terms of the statistical distribution of the channel power gains and of the Packet Error Rate (PER) curve of the common modulation and error correcting code employed by all users. Using Variational Calculus, we obtain a closed-form expression for the optimum energy profile at low average $E_{s} / N_{0}$ and prove that the aggregate spectral efficiency converges to a non-vanishing value if the user population is large and terminals implement an uncoordinated admission control based on the known PER curve.
\end{abstract}

\section{INTRODUCTION}

The application of successive interference cancellation (SIC) schemes to random access in scenarios subject to user power unbalance or power randomization [1] leads to significant performance gains in terms of the aggregate spectral efficiency. In particular, SIC lies at the core of Enhanced Spread Spectrum Aloha (E-SSA), which was developed in the context of uncoordinated random access for satellite communications [2][3] and is extensible to any random multipleaccess scenario dealing with a large number of low-rate users. Thus, a number of theoretical tools have been developed for performance evaluation and optimization of such schemes. A heuristic framework has been considered in [4] for performance optimization of SIC receivers in spread-spectrum multiple access networks. In the same context, it has been shown that Variational Calculus (VC) techniques are a valuable design tool for the determination of the optimum energy allocation under different SIC policies when constraints such as the users average energy [5] or a given power unbalance [6] are enforced.

Along these lines, this paper pursues to apply VC techniques to the determination of the optimum energy allocation rule in scenarios in which the users' transmitted energy is severely limited and thus noise dominates in front of multiple access interference after de-spreading, a fact that compromises the proven effectivity of SIC strategies in other operational regimes. For conciseness, we will refer to this operational regime as low average $E_{s} / N_{0}$. For a number of reasons, this scenario is of interest in machine-to-machine communications involving a massive number of devices communicating with a central receiver: firstly, in terms of energy consumption at both network and device level, it is convenient to limit transmission energy; secondly, in terms of co-channel or outof-band interference on other neighbouring services, it is also beneficial to reduce the average energy transmitted into the channel by the whole machine network. In addition, the consideration of the low average $E_{s} / N_{0}$ regime simplifies the mathematical difficulties associated with the analysis of iterative SIC receivers [7], and allows to predict accurately the asymptotic behaviour of other intricate multiuser detection schemes when the users available power gradually shrinks. In particular, it is shown that a non-vanishing aggregate spectral efficiency can be attained at very low average $E_{s} / N_{0}$ provided that the user population is large and the number of simultaneous users in the system is limited according to the known PER curve.

\section{SySTEM MODEL}

Let us consider a very large population of $K$ low-rate terminals that are connected to the same receiver. Let $h_{n}[k]$ denote the channel power gain between user $k$ and the receiver at time slot $n$. We consider that the $h_{n}[k]$ are identically distributed random variables of known probability distribution $F_{H}(h) \doteq \operatorname{Pr}(H<h)$ and that $h_{n}[k]$ and $h_{n^{\prime}}\left[k^{\prime}\right]$ are independent for any $k^{\prime} \neq k$ and slowly time-varying for $n^{\prime} \neq n$. Without loss of generality, users are re-indexed in every slot $n$ according to their instantaneous channel power gain $h_{n}[k]$ as follows: $h_{n}[1] \geq h_{n}[2] \geq \ldots \geq h_{n}[K]$.

Let $E_{n}[k]$ denote the transmitted energy per symbol of user $k$ at time slot $n$. As studied in [7], there exist two alternative ways for setting $\left.E_{n}[k]: 1\right)$ Random energy allocation: $E_{n}[k]$ is randomly selected from a continuous probability distribution $F_{E}(E)$; 2) Deterministic energy allocation: $E_{n}[k]=g\left(h_{n}[k]\right)$ with $g(h)$ a energy allocation function to be optimized. In the deterministic case, each user needs to know its channel gain $h_{n}[k]$, that can be estimated, assuming reciprocity, from the downlink signal as long as the channel coherence time is sufficiently long. Focusing on this scenario, the objective of this paper is to determine the uncoordinated energy allocation function $g(h)$ that maximizes the system performance when the average transmitted energy of every user is limited to $\bar{E}$ Joules per symbol:

$$
\lim _{n \rightarrow \infty} \frac{1}{n} \sum_{i=1}^{n} E_{i}[k]=\bar{E}
$$

Moreover, in the case of identically-distributed random channels, the temporal average in (1) coincides with the average 
over the $K$ users and, therefore, we have that

$$
\lim _{K \rightarrow \infty} \frac{1}{K} \sum_{j=1}^{K} E_{n}[j]=\bar{E}
$$

When the number of users is very large $(K \rightarrow \infty)$, it is convenient to normalize the user index $k$ with respect to $K$ and introduce the continuous user index $t \doteq k / K$. Then, in every time slot $n$, the channel gain $h_{n}[t K]$ of user $k=t K$ is known to converge asymptotically as $K \rightarrow \infty$ to

$$
h(t) \doteq \lim _{K \rightarrow \infty} h_{n}[t K]=F_{H}^{-1}(1-t)
$$

which is the channel power gain profile or quantile function [7]. In that case, the average energy constraint (2) reduces to

$$
\int_{0}^{1} E(t) \mathrm{d} t=\bar{E}
$$

where

$$
E(t) \doteq \lim _{K \rightarrow \infty} E_{n}[t K]=g(h(t))
$$

stands for the transmitted symbol energy of user $k=t K$. As there is a one-to-one mapping ${ }^{1}$ between $E(t)$ and $g(h)$, we can optimize the system performance over $E(t)$ and determine next the associated energy allocation function $g(h)$.

In a conventional single-user receiver, the Signal-toInterference-plus-Noise Ratio (SINR) of user $k$ after despreading, at time slot $n$, is given by:

$$
\Gamma_{n}[k]=\frac{E_{n}[k] h_{n}[k]}{N_{0}+\frac{1}{N} \sum_{j \neq k} E_{n}[j] h_{n}[j]}
$$

with $N_{0}$ the noise power spectral density and $N$ the spreading factor. The above formula also corresponds to the SINR of the first stage of a SIC receiver for $k=1$. For subsequent stages of the SIC receiver $(k>1)$, or for any other multiuser receiver architecture, the interference in the denominator is lower and (6) constitutes then a lower bound of the SINR (worst case).

In the asymptotic case $(K \rightarrow \infty)$, introducing again the continuous index $t=k / K$ and defining $\bar{E}(t) \doteq E(t) / \bar{E}$, the SINR of user $k=t K$ in equation (6) tends to

$$
\Gamma(t) \doteq \lim _{K \rightarrow \infty} \Gamma_{n}[t K]=\frac{E(t) h(t)}{N_{0}+\alpha \bar{E} \int_{0}^{1} \bar{E}(\tau) h(\tau) \mathrm{d} \tau}
$$

with $\alpha \doteq K / N$ the constant system load.

In this paper, we consider that the users average transmitted energy is very low (i.e., $\bar{E} / N_{0} \ll 1$ ) and that, relative to noise, the interference term in the denominator of (7) is negligible. Consequently, whichever the single- or multi-user receiver we consider, the SINR in (7) converges at low average $E_{s} / N_{0}$ to

$$
\Gamma(t)=\frac{E(t) h(t)}{N_{0}}+o\left(\frac{\bar{E}}{N_{0}}\right)
$$

using Landau's little "o" notation for $\bar{E} / N_{0} \rightarrow 0$.

\footnotetext{
${ }^{1}$ For a given $E(t)$, the corresponding energy allocation function is $g(h)=$ $\tilde{E}\left(F_{H}(h)\right)$ with $E(t) \doteq E(1-t)$ the reversed version of $E(t)$.
}

In this low average $E_{s} / N_{0}$ regime, the system load $\alpha$ must fulfil the following equation:

$$
\alpha=c \frac{N_{0} / \bar{E}}{\int_{0}^{1} \bar{E}(t) h(t) \mathrm{d} t}
$$

with $c \ll 1$ a small constant determining the receiver interference-to-noise ratio (INR). Therefore, for a fixed $c$ value, the system load $\alpha$ is proportional to the inverse of $\bar{E} / N_{0}$.

We consider for simplicity that all users have a common physical layer (i.e., modulation and coding), which is characterized by the Packet Success Rate (PSR) versus SINR curve: $\operatorname{PSR}(\Gamma) \doteq 1-\operatorname{PER}(\Gamma)$. Thus, the aggregate spectral efficiency (bps/Hz) of the system at low $E_{s} / N_{0}$ reads

$$
S E=\frac{R_{c}}{N(1+\beta)} \sum_{k=1}^{K} \operatorname{PSR}\left(\frac{E_{n}[k] h_{n}[k]}{N_{0}}\right)
$$

with $N$ the spreading factor, $R_{c}$ the number of information bits per symbol and $\beta$ the roll-off factor of the shaping pulse. Asymptotically, with $K=\alpha N \rightarrow \infty$, the aggregate spectral efficiency in (10) converges to

$$
\lim _{K \rightarrow \infty} \mathrm{SE}=\frac{R_{c} \alpha}{1+\beta} \int_{0}^{1} \operatorname{PSR}\left(\frac{E(t) h(t)}{N_{0}}\right) \mathrm{d} t
$$

It is worth noting that the frequency flat fading assumption adopted so far remains reasonably valid for scenarios in which the system bandwidth $W$ is rather lower than the channel coherence bandwidth. Accordingly, in the asymptotic analysis we are considering implicitly that the symbol rate $R_{s}=W /(N(1+\beta))$ goes to zero as $N=K / \alpha \rightarrow \infty$.

Based on previous studies [5], it is convenient to implement an admission control procedure in order to cope with arbitrarily large system loads $\alpha$. To achieve this, only the strongest $K_{0}=t_{0} K$ users are allowed to transmit, with $t_{0} \doteq K_{0} / K$ an admission control parameter that needs to be optimized $\left(0<t_{0} \leq 1\right)$. In order to maximize (11), the admitted users are those with the highest received energy per symbol $E(t) h(t)$. If we assume that $E(t)$ is a monotonic function of $t$ that does not alter the order defined by $h(t)$ (as proved in Section III), the strongest users correspond to the first $t_{0}$ users $\left(0<t \leq t_{0}\right)$. The rest of users remain silent, i.e. $E(t)=0$ for $t_{0}<t \leq 1$. In that case, the optimization problem becomes

$$
\max _{0<t_{0} \leq 1} \max _{E(t)} \int_{0}^{t_{0}} \operatorname{PSR}\left(\frac{E(t) h(t)}{N_{0}}\right) \mathrm{d} t
$$

subject to

$$
\int_{0}^{t_{0}} E(t) \mathrm{d} t=\bar{E}
$$

Note that $t_{0}=1$ is always a candidate solution. Therefore, the introduction of $t_{0}$ does not reduce the set of available solutions.

\section{Optimum Energy Allocation Profile}

The constrained variational problem in equations (12)-(13) can be formulated using a Lagrange multiplier that incorporates the average energy constraint (13):

$$
\max _{0<t_{0} \leq 1} \max _{E(t)} \int_{0}^{t_{0}}\left[\operatorname{PSR}\left(\frac{E(t) h(t)}{N_{0}}\right)-\rho E(t)\right] \mathrm{d} t
$$


Following the standard procedure in Variational Calculus [8], if $E_{0}(t)$ stands for the optimum value of $E(t)$, an infinitesimal admissible $e^{2}$ variation $a \cdot v(t)$, with $a \rightarrow 0$, is applied to $E_{0}(t)$ along direction $v(t)$, obtaining the perturbed energy profile:

$$
E(t)=E_{0}(t)+a \cdot v(t)
$$

Then, a necessary condition for $E_{0}(t)$ to be an extremum of the inner optimization over $E(t)$ in (14) (for $t_{0}$ fixed) is that

$$
\lim _{a \rightarrow 0} \frac{\mathrm{d}}{\mathrm{d} a} \int_{0}^{t_{0}}\left[\operatorname{PSR}\left(\frac{E(t) h(t)}{N_{0}}\right)-\rho E(t)\right] \mathrm{d} t=0
$$

which, using standard differentiation rules, yields

$$
\int_{0}^{t_{0}}\left[\operatorname{PSR}^{\prime}\left(\frac{E_{0}(t) h(t)}{N_{0}}\right) \frac{h(t)}{N_{0}}-\rho\right] v(t) \mathrm{d} t=0
$$

As (17) must be satisfied for any admissible variation $v(t)$, the optimum $E_{0}(t)$ must verify the following invariance equation:

$$
\operatorname{PSR}^{\prime}\left(\frac{E_{0}(t) h(t)}{N_{0}}\right) h(t)=\lambda \quad 0<t \leq t_{0}
$$

with $\operatorname{PSR}^{\prime}(\Gamma) \doteq \frac{\mathrm{d}}{\mathrm{d} \Gamma} \mathrm{PSR}(\Gamma)$ the derivative of the PSR function and $\lambda$ a constant value. Additionally, $E_{0}(t)$ in (18) is a maximum of the spectral efficiency if the second derivative is negative for any non-null admissible variation $v(t)$ :

$$
\begin{aligned}
& \lim _{a \rightarrow 0} \frac{\mathrm{d}^{2}}{\mathrm{~d} a^{2}} \int_{0}^{t_{0}}\left[\operatorname{PSR}\left(\frac{E(t) h(t)}{N_{0}}\right)-\rho E(t)\right] \mathrm{d} t<0 \\
& \int_{0}^{t_{0}}\left[\operatorname{PSR}^{\prime \prime}\left(\frac{E_{0}(t) h(t)}{N_{0}}\right) \frac{h^{2}(t)}{N_{0}^{2}}\right] v^{2}(t) \mathrm{d} t<0
\end{aligned}
$$

It is not difficult to check that (19) requires that ${ }^{3}$

$$
\begin{gathered}
\mathrm{PSR}^{\prime \prime}\left(\frac{E_{0}(t) h(t)}{N_{0}}\right)<0 \quad 0<t \leq t_{0} \\
\frac{E_{0}(t) h(t)}{N_{0}}>\Gamma_{i p} \quad 0<t \leq t_{0}
\end{gathered}
$$

where $\operatorname{PSR}^{\prime \prime}(\Gamma) \doteq \mathrm{d}^{2} \operatorname{PSR}(\Gamma) / \mathrm{d}^{2}$ and $\Gamma_{i p}$ is the inflexion point of the PSR curve, i.e., $\operatorname{PSR}^{\prime \prime}\left(\Gamma_{i p}\right)=0$. As shown later, the last condition is satisfied in case of practical modulation and coding schemes. In that case, the optimum energy allocation profile $E_{0}(t)$ can be derived from (18). Introducing the definition $\varphi(x) \doteq \mathrm{PSR}^{\prime-1}(x)$ to compact the notation, it follows that

$$
E_{0}(t)=\left\{\begin{array}{cc}
\frac{N_{0}}{h(t)} \varphi\left(\frac{\lambda}{h(t)}\right) & 0<t \leq t_{0} \\
0 & t_{0}<t \leq 1
\end{array}\right.
$$

which is valid for any value of $\lambda$ in (22) for which the inverse of $\operatorname{PSR}^{\prime}(\Gamma)$ exists, i.e.,

$$
0<\lambda \leq h\left(t_{0}\right) \mathrm{PSR}_{\max }^{\prime}
$$

\footnotetext{
${ }^{2} \mathrm{~A}$ variation $v(t)$ is admissible if $E(t)=E_{0}(t)+a v(t)$ satisfies the average energy constraint (4) for non-null infinitesimal values of $a$. Computing the derivative of (4) with respect to $a$ and taking the limit for $a \rightarrow 0$, we find that the admissible variations are those satisfying $\int_{0}^{t_{0}} v(t) \mathrm{d} t=0$.

${ }^{3}$ If (20)-(21) were not satisfied, (19) would not hold for those admissible variations $v(t)$ that are null at $t \in \mathcal{T} \doteq\left\{t: \mathrm{PSR}^{\prime \prime}\left(E_{0}(t) h(t) / N_{0}\right)<0\right\}$ and non-null at some other $t \notin \mathcal{T}$ for which $\int_{t \notin \mathcal{T}} v(t) d t=0$.
}

with $\operatorname{PSR}_{\text {max }}^{\prime} \doteq \max _{\Gamma}\left\{\operatorname{PSR}^{\prime}(\Gamma)\right\}=\operatorname{PSR}^{\prime}\left(\Gamma_{i p}\right)$. Inside the interval above, the value of $\lambda$ is the one satisfying the average energy constraint (13) and, therefore, $\lambda=\lambda\left(t_{0}\right)$ is an implicit function of the admission control parameter $t_{0}$ (see Appendix).

From (22), it is manifest that the energy allocation profile at reception $E_{0}(t) h(t)$ is always a non-increasing ${ }^{4}$ function of $t$ that does not alter the order of users defined by $h(t)$, as it was assumed in Sec. II. In particular, at low $E_{s} / N_{0}$, the optimum SINR profile (8) is the following non-increasing function of $t$ :

$$
\Gamma_{0}(t)=\frac{E_{0}(t) h(t)}{N_{0}}=\left\{\begin{array}{cc}
\varphi\left(\frac{\lambda\left(t_{0}\right)}{h(t)}\right) & 0<t \leq t_{0} \\
0 & t_{0}<t \leq 1
\end{array}\right.
$$

The energy and SINR profiles in (22) and (24) are therefore functions of the admission parameter $t_{0}$. To determine the optimum $t_{0}$, we must substitute (24) into (12) and optimize then with respect to $t_{0}$ :

$$
t_{\text {opt }}=\underset{0<t_{0} \leq 1}{\operatorname{argmax}} \int_{0}^{t_{0}} \operatorname{PSR}\left(\varphi\left(\frac{\lambda\left(t_{0}\right)}{h(t)}\right)\right) \mathrm{d} t
$$

For $t_{\mathrm{opt}}<1$, it is found in the Appendix that $t_{\mathrm{opt}}$ coincides with the value of $t_{0}$ fixing the SINR of the weakest admitted user, i.e. $\Gamma_{0}\left(t_{0}\right)$ in (24), to the following value:

$$
\Gamma^{*} \doteq \arg \max _{\Gamma} \operatorname{PSR}(\Gamma) / \Gamma
$$

which is characteristic of the adopted modulation and coding scheme. Moreover, we have checked that $\Gamma^{*}>\Gamma_{i p}$ in case of practical modulation and coding schemes and, therefore, the extremality condition in (21) is always verified in practice.

Finally, incorporating (24) and (25) into (11), we obtain the following expression for the aggregate spectral efficiency ${ }^{5}$ :

$$
\lim _{K \rightarrow \infty} \mathrm{SE}=\frac{R_{c} \alpha}{1+\beta} \int_{0}^{t_{\mathrm{opt}}} \operatorname{PSR}\left(\varphi\left(\frac{\lambda\left(t_{\mathrm{opt}}\right)}{h(t)}\right)\right) \mathrm{d} t
$$

which is valid for any fixed load factor $\alpha$ that fulfils (9).

If the system load $\alpha$ is not fixed but it is adapted to the working $E_{s} / N_{0}$ as in (9), the aggregate spectral efficiency converges asymptotically as $K \rightarrow \infty$ to the following expression:

$$
\mathrm{SE}_{\mathrm{adpt}}=\frac{c R_{c}}{1+\beta} \frac{\int_{0}^{t_{\mathrm{opt}}} \operatorname{PSR}\left(\varphi\left(\frac{\lambda\left(t_{\mathrm{opt}}\right)}{h(t)}\right)\right) \mathrm{d} t}{\int_{0}^{t_{\mathrm{opt}}} \varphi\left(\frac{\lambda\left(t_{\mathrm{opt}}\right)}{h(t)}\right) \mathrm{d} t},
$$

that is achieved by limiting the instantaneous system load to

$$
\alpha_{0} \doteq K_{0} / N=\alpha t_{\mathrm{opt}}=\frac{c t_{\mathrm{opt}}}{\int_{0}^{t_{\mathrm{opt}}} \varphi\left(\frac{\lambda\left(t_{\mathrm{opt}}\right)}{h(t)}\right) \mathrm{d} t}
$$

Load regulation (9) allows the aggregate spectral efficiency (28) not to vanish as $\bar{E} / N_{0} \rightarrow 0$. In fact, taking into account that $\lim _{\bar{E} \rightarrow 0} \Gamma_{0}(t)=\Gamma^{*}$ for $0<t \leq t_{\text {opt }}$ irrespectively of the channel power gain profile $h(t)$ (Appendix), we conclude that

$$
\begin{gathered}
\lim _{\bar{E} \rightarrow 0} \mathrm{SE}_{\mathrm{adpt}}=\frac{c R_{c}}{1+\beta} \frac{\operatorname{PSR}\left(\Gamma^{*}\right)}{\Gamma^{*}} \\
\lim _{\bar{E} \rightarrow 0} \alpha_{0}=c / \Gamma^{*}
\end{gathered}
$$

\footnotetext{
${ }^{4}$ If (21) holds, $\varphi(x)$ is a decreasing function of $x$ in the interval $0<x<$ $\mathrm{PSR}_{\text {max }}^{\prime}$. Moreover, its argument $x=\frac{\lambda\left(t_{0}\right)}{h(t)}$ is a non-decreasing function of $t$ since $h(t)$ is non-increasing and $\lambda\left(t_{0}\right)$ is constant in $t$.

${ }^{5}$ If $t_{\mathrm{opt}}<1, \lambda\left(t_{\mathrm{opt}}\right)=h\left(t_{\mathrm{opt}}\right) \mathrm{PSR}^{\prime}\left(\Gamma^{*}\right)$, as proved in the Appendix.
} 


\section{CLOSED-FORM RESULTS: THE CONSTANT CHANNEL}

We consider the asymptotic case in which the power unbalance $\log (h(0) / h(1))$ induced by the channel is very small and all users share approximately the same channel gain $h$. Substituting $h(t)=h$ into (22), it follows that the optimum energy profile $\frac{N_{0}}{h} \varphi\left(\frac{\lambda}{h}\right)$ is constant for $0<t \leq t_{0}$ and, to fulfil the energy constraint in (13), equal to $\bar{E} / t_{0}$ over that interval. Thus, the optimum energy profile in (22) reduces to

$$
E_{0}(t)=\left\{\begin{array}{cc}
\bar{E} / t_{0} & 0<t \leq t_{0} \\
0 & t_{0}<t \leq 1
\end{array}\right.
$$

which is a function of $t_{0}$. If we plug now (32) into (12), or alternatively into (25), we obtain that

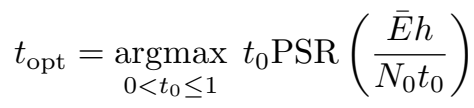

In order to find $t_{\mathrm{opt}}$, we reformulate (33) in terms of the users common SINR (8), which is now given by:

$$
\Gamma=\frac{\bar{E} h}{N_{0} t_{0}}
$$

From (34), we may express $t_{0}$ versus $\Gamma$. Then, the maximization in (33) over $t_{0}$ is found equivalent to the maximization in (26) over $\Gamma$. Thus, $t_{\mathrm{opt}}$ is the value of $t_{0}$ enforcing the SINR of all users to $\Gamma^{*}(26)$. Solving for $t_{0}$ in (34) and taking into account that $t_{0} \leq 1$, we get:

$$
t_{\mathrm{opt}}=\left\{\begin{array}{cl}
\frac{\bar{E} h}{N_{0} \Gamma^{*}} & \frac{\bar{E} h}{N_{0}}<\Gamma^{*} \\
1 & \frac{\bar{E} h}{N_{0}} \geq \Gamma^{*}
\end{array}\right.
$$

Note that this value of $t_{0}$ guarantees that the SINR of any user never falls below $\Gamma^{*}$, even if the average transmitted energy $\bar{E}$ is very low. Plugging (35) into (34), this is easily shown by:

$$
\Gamma_{\mathrm{opt}} \doteq \frac{\bar{E} h}{N_{0} t_{\mathrm{opt}}}= \begin{cases}\Gamma^{*} & \frac{\bar{E} h}{N_{0}} \leq \Gamma^{*} \\ \frac{\bar{E} h}{N_{0}} & \frac{\bar{E} h}{N_{0}}>\Gamma^{*}\end{cases}
$$

If we plug (32) and (35) into (12) and fix the system load to $\alpha$, we obtain that the aggregate spectral efficiency is given by

$$
\lim _{K \rightarrow \infty} \mathrm{SE}=\frac{R_{c} \alpha}{1+\beta} \frac{\bar{E} h}{N_{0}} \frac{\operatorname{PSR}\left(\Gamma_{\mathrm{opt}}\right)}{\Gamma_{\mathrm{opt}}}
$$

Alternatively, if $\alpha$ is regulated as in (9), we obtain that

$$
\mathrm{SE}_{\mathrm{adpt}}=\frac{c R_{c}}{1+\beta} \frac{\operatorname{PSR}\left(\Gamma_{\mathrm{opt}}\right)}{\Gamma_{\mathrm{opt}}}
$$

In the last equation, the load is set to $\alpha=c N_{0} /(\bar{E} h)$ according to (9) and, therefore, $\alpha$ is inversely proportional to $\bar{E} / N_{0}$. This load regulation policy allows the aggregate spectral efficiency not to collapse as $\bar{E} / N_{0} \rightarrow 0$. In particular, if we take into account that $\lim _{\bar{E} \rightarrow 0} \Gamma_{\text {opt }}=\Gamma^{*}$ (36), it follows that the aggregate spectral efficiency $\mathrm{SE}_{\text {adpt }}$ and the instantaneous load $\alpha_{0}=K_{0} / N$ converge to (30) and (31), respectively.

To sum up, the following random access is optimum when the average $E_{s} / N_{0}$ is very low $\left(\bar{E} / N_{0}<\Gamma^{*} / h\right)$, the channel is constant and the number of users is large $(K \rightarrow \infty)$ : users transmit with probability $t_{\text {opt }}$ (35) and remain silent with probability $1-t_{\text {opt }}$, with $t_{\text {opt }}$ proportional to $\bar{E} / N_{0}$. During silence intervals, users accumulate energy to guarantee the received SINR is not lower than $\Gamma^{*}(26)$ during the activity

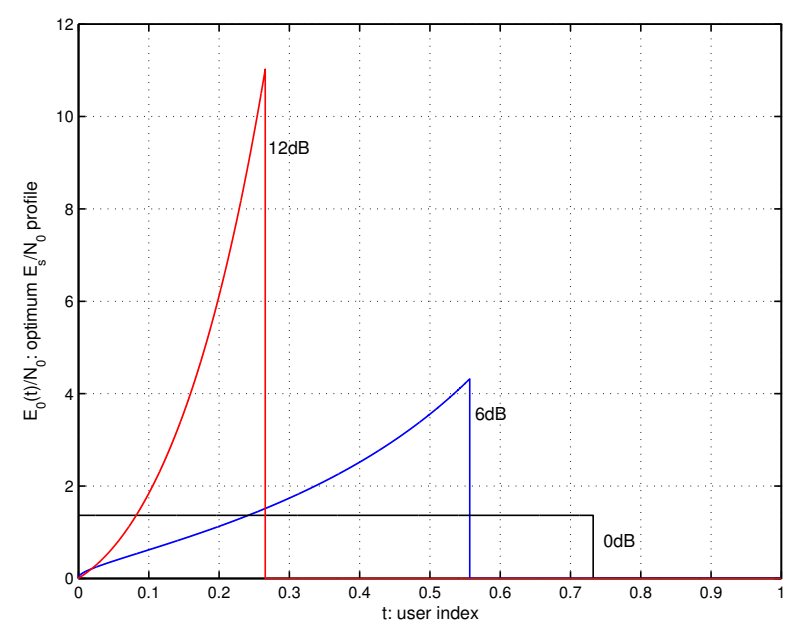

Fig. 1. Optimum Energy profile for different values of $\sigma$.

intervals. To compensate for the reduced per-user efficiency $t_{\text {opt }} \operatorname{PSR}\left(\Gamma^{*}\right)$, the system load $\alpha$ is increased in proportion to $N_{0} / \bar{E}$ while the instantaneous load $\alpha_{0}$ is set to the constant value $c / \Gamma^{*}$. Another relevant feature of the proposed random access scheme is that the transmitted peak power is limited to $R_{s} N_{0} \Gamma^{*} / h$ watts with $R_{s}=W /(N(1+\beta))$ the asymptotically vanishing symbol rate.

\section{NUMERICAL RESULTS: THE LOG-NORMAL CHANNEL}

In order to validate the analytical results of the paper, we consider in this section a log-normal distribution $\ln (H) \sim$ $\mathcal{N}\left(\mu, \sigma^{2}\right)$ for the users channel gain $H$, with $\mu$ and $\sigma$ the mean and standard deviation of the logarithmic channel power gain, respectively. In this case, the channel profile $h(t)$ reads

$$
h(t)=F_{H}^{-1}(1-t)=\exp \left(\mu+\sigma Q^{-1}(t)\right)
$$

with $Q^{-1}(t)$ the inverse of $Q(y)=\frac{1}{\sqrt{2 \pi}} \int_{y}^{\infty} e^{-x^{2} / 2} \mathrm{~d} x$ (Qfunction). For simplicity, we fix the average channel power gain $\mathbb{E}\{H\}=\int_{0}^{1} h(t) \mathrm{d} t=\exp \left(\mu+\sigma^{2} / 2\right)$ to 1 and test different values of $\sigma$. Note that $\sigma=0$ corresponds to the constant channel case addressed in Section IV.

For the physical layer, we consider QPSK symbols and the standardized DVB-RCS Turbo Code of rate 1/2 [9]. Anyway, the results in this section can be replicated for any modulation and coding scheme as long as we know its $\operatorname{PSR}(\Gamma)$ curve.

In Fig. 1, we show the optimum transmitted $E_{s} / N_{0}$ profile $E_{0}(t) / N_{0}$ for a working $E_{s} / N_{0}$ of $0 \mathrm{~dB}\left(\bar{E} / N_{0}=1\right)$, and three values of $\sigma: 0 \mathrm{~dB}, 6 \mathrm{~dB}$ and $12 \mathrm{~dB}$. It is shown that the optimum energy allocation policy partially compensates for the channel power unbalance while limiting the number of active transmitters $\left(t_{\text {opt }}<1\right)$. As a result, Fig. 2 shows that the reception SINR $\Gamma(t)$ is a decreasing function of $t$ for $\sigma>0$ whereas $\Gamma(t)$ converges to constant $\Gamma^{*}(26)$ when $\sigma=0$. In Fig. 2, it is also evidenced that the SINR of the weakest user is set to $\Gamma^{*}$ independently of $h(t)$, as proved in the Appendix.

Fig. 3 illustrates the applicability of the paper to predict the asymptotic behaviour of distinct SIC architectures at low average $E_{s} / N_{0}$. In particular, Fig. 3 depicts the $E_{s} / N_{0}$ profile that maximizes the aggregate spectral efficiency of a conventional SIC receiver [7], at arbitrary $E_{s} / N_{0}$ and system load 


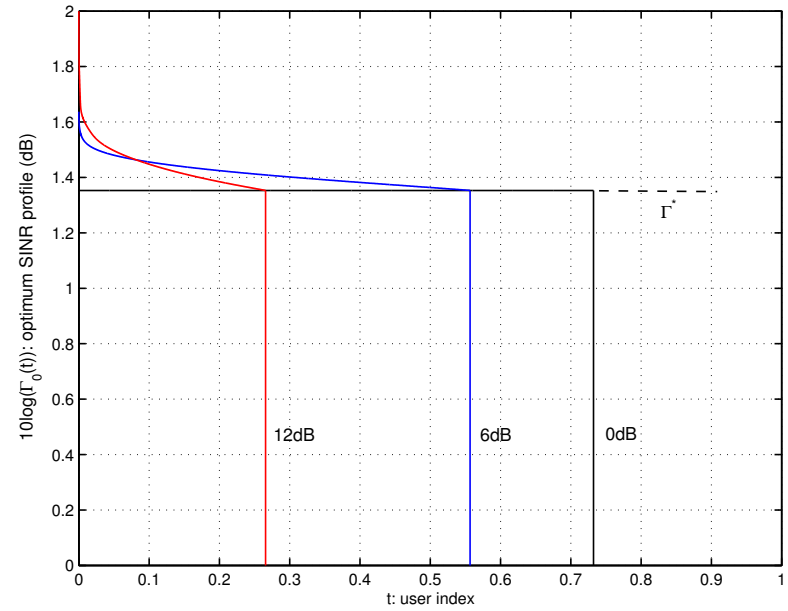

Fig. 2. Optimum SINR profile for different values of $\sigma$.

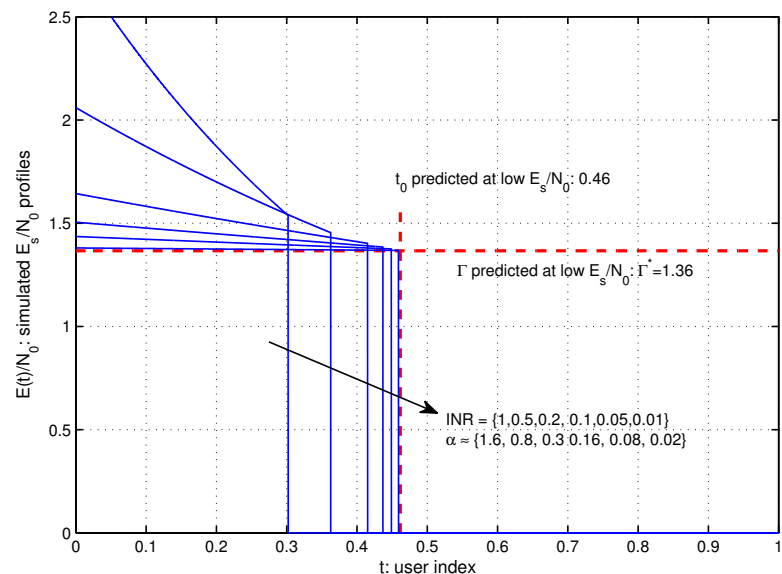

Fig. 3. Convergence of the optimum $E_{s} / N_{0}$ profile (assuming uniform SINR) as the interference-to-noise ratio (INR) decreases. A constant channel is considered, as in Section IV.

$\alpha$, when the SINR of decoded users is kept constant: $\Gamma(t)=\Gamma$ $\left(0<t \leq t_{0}\right)$. Simulations are carried out for an average $E_{s} / N_{0}$ of $-2 \mathrm{~dB}\left(\bar{E} / N_{0}=0.631\right)$ and different interference-tonoise ratios (INR). As in Section IV, we consider that $h(t)$ is constant $(\sigma=0)$. Horizontal and vertical dotted lines indicate the predicted $t_{\mathrm{opt}}(35)$ and $\Gamma_{\mathrm{opt}}(36)$ at low average $E_{s} / N_{0}$. As shown in Fig. 3, when the INR decreases, the $E_{s} / N_{0}$ profile of the studied SIC architecture tends to the energy profile in (32) as long as the interference becomes negligible compared to the noise. It is worth noting that this asymptotic result is rather general and applies to any other multiuser schemes maximizing the aggregate spectral efficiency.

\section{APPENDIX: DETERMINATION OF $t_{\mathrm{opt}}<1$.}

Using (22), the average energy constraint $\int_{0}^{t_{0}} E_{0}(t) \mathrm{d} t=\bar{E}$ defines an implicit function $\lambda=\lambda\left(t_{0}\right)$, written as

$$
\phi\left(t_{0}, \lambda\right) \doteq-\bar{E}+\int_{0}^{t_{0}} E_{0}(t) \mathrm{d} t=0
$$

Let $J\left(t_{0}\right)$ be the integral in (25) that has to be maximized. A necessary condition for $t_{\mathrm{opt}}<1$ to yield an extremum is that

$$
\begin{aligned}
& \frac{\mathrm{d}}{\mathrm{d} t_{0}} J\left(t_{0}\right)=0 \text { at } t_{0}=t_{\mathrm{opt}} \text {. Hence, } \\
& \operatorname{PSR}\left(\Gamma_{0}\left(t_{\mathrm{opt}}\right)\right)+\int_{0}^{t_{\mathrm{opt}}} \frac{\lambda\left(t_{\mathrm{opt}}\right)}{h(t)} \varphi^{\prime}\left(\frac{\lambda\left(t_{\mathrm{opt}}\right)}{h(t)}\right) \frac{\lambda^{\prime}\left(t_{\mathrm{opt}}\right)}{h(t)} \mathrm{d} t=0
\end{aligned}
$$

where we have used the expression for $\Gamma_{0}(t)$ in (24). The derivative $\lambda^{\prime}\left(t_{0}\right)$ can be obtained from the Implicit Function Theorem as $\lambda^{\prime}\left(t_{0}\right)=-\frac{\partial}{\partial t_{0}} \phi / \frac{\partial}{\partial \lambda} \phi$, which yields,

$$
\lambda^{\prime}\left(t_{0}\right)=-\frac{N_{0}}{h\left(t_{0}\right)} \varphi\left(\frac{\lambda\left(t_{0}\right)}{h\left(t_{0}\right)}\right)\left(\int_{0}^{t_{0}} \frac{N_{0}}{h^{2}(t)} \varphi^{\prime}\left(\frac{\lambda\left(t_{0}\right)}{h(t)}\right) \mathrm{d} t\right)^{-1}
$$

Plugging (39) into (38) and using (24) again, some algebra shows that (38) reduces to $\operatorname{PSR}\left(\Gamma_{0}\left(t_{\text {opt }}\right)\right)=\operatorname{PSR}^{\prime}\left(\Gamma_{0}\left(t_{\text {opt }}\right)\right)$. $\Gamma_{0}\left(t_{\text {opt }}\right)$. It is straightforward that the solution to this nonlinear equation is $\Gamma_{0}\left(t_{\mathrm{opt}}\right)=\Gamma^{*}$, the maximizing $\Gamma^{*}$ in (26).

If $t_{\mathrm{opt}}<1, \lambda\left(t_{\mathrm{opt}}\right)$ can be obtained explicitly by imposing $\Gamma_{0}\left(t_{\text {opt }}\right)=\Gamma^{*}$ in (24), yielding $\lambda\left(t_{\text {opt }}\right)=h\left(t_{\text {opt }}\right) \operatorname{PSR}^{\prime}\left(\Gamma^{*}\right)$. Finally, $t_{\text {opt }}$ is obtained by solving $\phi\left(t_{\text {opt }}, \lambda\left(t_{\text {opt }}\right)\right)=0$. If this equation yields a $t_{\mathrm{opt}}$ larger than 1 , then $t_{\mathrm{opt}}$ is set to 1 .

Next we prove that $\lim _{\bar{E} \rightarrow 0} \Gamma_{0}(t)=\Gamma^{*}$. Using (23) in (22), it follows that $E_{0}(t)>N_{0} \Gamma_{i p} / h\left(t_{\mathrm{opt}}\right)$ for $0<t<t_{\mathrm{opt}}$. Thus, if $\bar{E} \rightarrow 0, \int_{0}^{t_{\mathrm{opt}}} E_{0}(t) \mathrm{d} t=\bar{E}$ requires that $t_{\mathrm{opt}} \rightarrow 0$. As $t_{\text {opt }}<1$, we can substitute $\lambda\left(t_{\text {opt }}\right)=h\left(t_{\text {opt }}\right) \operatorname{PSR}^{\prime}\left(\Gamma^{*}\right)$ into (24) and compute the following $\operatorname{limit} \lim _{t_{\mathrm{opt}} \rightarrow 0} \Gamma_{0}(t)=\Gamma^{*}$.

\section{CONCLUSIONS}

This paper has derived the energy distribution of the transmitting users that optimizes the aggregate spectral efficiency of a massive spread-spectrum network operating at low average $E_{s} / N_{0}$ : that is, when multiple-access interference is negligible with respect to thermal noise. Under this assumption, the peruser SINR expression is substantially simplified and allows to tackle the design of the optimum energy profile for a given channel power gain profile. It is proved then that the aggregate spectral efficiency does not vanish at low average $E_{s} / N_{0}$, whatever the channel gain profile, as long as the system load $\alpha$ is adjusted according to the working average $E_{s} / N_{0}$. Rather, the admission control rule built into the energy allocation scheme warrants that all the active users operate at an optimum SINR that depends uniquely on the specific PER versus SINR characteristic curve. Thus, the paper analysis allows to better understand the behaviour of multiuser detection schemes, which exhibit two different regimes: a first regime at mediumto-high average $E_{s} / N_{0}$ without admission control (all users are active) and a second regime at low and very low average $E_{s} / N_{0}$ featuring admission control (some users remain temporarily silent saving energy for the next transmission). The main contribution of this paper is the characterization of this second regime which is of interest for massive lowconsumption machine-to-machine communications.

\section{ACKNOWLEDGMENT}

This work has been jointly supported by projects: (i) TEC2013-47020-C2-2-R (COMPASS), Ministerio de Economía y Comptetitividad, Spanish National Research Plan; (ii) 2014 SGR 60 AGAUR (Catalan government). The authors would also like to thank the anonymous reviewers for their valuable comments and suggestions to improve the quality of the paper. 


\section{REFERENCES}

[1] P. Salvo Rossi, K. Kansanen, R. Muller, and C. Rachinger, "Power randomization for iterative detection over random-access fading channels," IEEE Transactions on Wireless Communications, vol. 14, no. 10, pp. 5704-5713, Oct 2015.

[2] O. D. R. Herrero and R. D. Gaudenzi, "High efficiency satellite multiple access scheme for machine-to-machine communications," IEEE Transactions on Aerospace and Electronics Engineering, vol. 48, no. 4, pp. 2961 - 2989, October 2012.

[3] G. Gallinaro, N. Alagha, R. De Gaudenzi, K. Kansanen, R. Muller, and P. Rossi, "ME-SSA: An advanced random access for the satellite return channel," in 2015 IEEE International Conference on Communications (ICC), June 2015, pp. 856-861.

[4] F. Collard and R. De Gaudenzi, "On the optimum packet power distribution for spread Aloha packet detectors with iterative successive interference cancelation," IEEE Transactions on Wireless Communications, vol. 13, no. 12, pp. 6783-6794, Dec 2014.

[5] J. Sala-Alvarez, J. Villares, and F. Rey, "SINR profile for spectral efficiency optimization of SIC receivers in the many-user regime," in Proc. IEEE ICC 2015 Workshop on Massive Uncoordinated Access Protocols (MASSAP'15), London, U.K., Jun. 2015.

[6] J. Sala, F. Rey, and J. Villares, "Asymptotically optimum energy profile for successive interference cancellation in DS-CDMA under a power unbalance constraint," IEEE Communications Letters, vol. 16, no. 2, pp. 172 - 175, February 2012.

[7] D. Djonin and V. K. Bhargava, "Asymptotic analysis of the conventional decision feedback receiver in fading channels," IEEE Transactions on Wireless Communications, vol. 2, no. 5, pp. 1066 - 1078, May 2003.

[8] D. Elsgolc, Calculus of Variations. Dover Publications, 2007.

[9] "Digital video broadcasting (DVB); interaction channel for satellite distribution systems," ETSI EN 301790 V1.2.2 (2000-12) European Telecommunications Standards Institute (ETSI). 